\title{
Simultaneous inversion of multiples and primaries: Inversion versus subtraction
}

\author{
Y, Luo and Panos G. Kelamis, Saudi Aramco, Dhahran, Saudi Arabia \\ Yanghua Wang, Robertson Research, Kent, England, U.K.
}

$I_{n}$ the this article, we propose an inversion scheme to replace the adaptive subtraction approaches which are widely used in conventional two-step (prediction + subtraction) multiple elimination methods. This new method, named SIMP (simultaneous inversion for multiples and primaries), inverts seismic data using constraints (e.g., modeled multiples and/or geologic discriminants) for multiples and primaries simultaneously. SIMP incorporates pattern recognition and shaping filters into one concise and practically solvable formulation. Its main advantages are that no orthogonality between multiples and primaries is assumed and that wavelet information is not necessary for the inversion.

Figure 1 compares conventional prediction plus subtraction and the SIMP approaches. In the conventional method (Figure 1a), predicted multiples are generated first, the shaping filters are derived second, and finally the primary component is obtained by subtracting the multiples shaped by the filters from the input data. In one SIMP procedure (SIMP I, Figure 1b), the predicted multiples are generated first, but the shaping filters (not the multiples) and the primaries are inverted simultaneously by a constrained inversion approach. A general SIMP approach (SIMP II, Figure 1c) inverts for multiples and primaries simultaneously assuming proper constraints are available.

Modern multiple elimination methodologies are firmly rooted in the principles of wave theory. In general, these multiple removal schemes involve two basic steps: multiple prediction and adaptive subtraction. The multiple prediction can be model-driven or data-driven. During the prediction step, the kinematic part of the multiples (traveltimes) is obtained. The adaptive subtraction step aims to obtain the dynamic part of the multiples (e.g., amplitudes and waveform shaping).

Once multiple-model traces are predicted, based on the original seismic data and/or a subsurface model, they need to be "shaped" in order to "match" the actual multiples. This is usually achieved with an adaptive least-squares subtraction approach and the matching filter may be represented as

$$
p(t)=y(t)-\sum_{j=1}^{N} f_{j}(t) * m_{j}(t)
$$

where $\mathrm{y}(\mathrm{t})$ is a raw data trace, $\mathrm{m}(\mathrm{t})$ are modeled multiple traces, $\mathrm{N}$ is the number of channels involved in matching, $f(t)$ are operators for adapting the group of $N$ traces $m(t)$ to the desired output $y(t),{ }^{*}$ indicates convolution, and $p(t)$ is the matching residual (primaries).

The filter coefficients, $f(t)$, are calculated using the wellknown minimum-energy criterion. The fundamental problem with minimizing the energy of the primary $p(t)$ is the assumption that multiples and primaries in the data are orthogonal. This assumption will be violated in most field

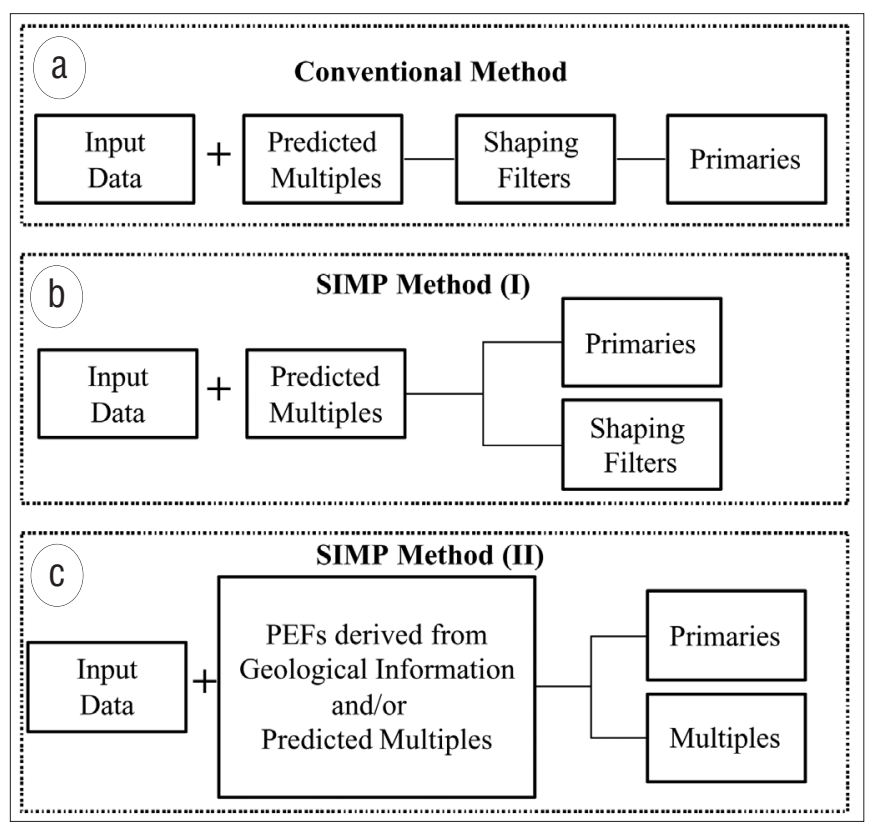

Figure 1. Block diagram of conventional method (a) and SIMP methods $(b$ and $c)$.

data sets and, consequently, primaries overlapping modeled multiples will be wrongly subtracted from the data. Thus, a "leakage" will always be present. To overcome this problem, Spitz (1999) proposed a "pattern recognition" subtraction based on prediction error filters (PEF).

In the following, we start from shaping filtering concepts and develop a concise formulation that does not require the orthogonal assumption. After that, a more general formula is developed to estimate multiples and primaries by using PEF, instead of explicitly modeled multiples. This is similar to Spitz's pattern recognition work but has the flexibility to incorporate more constraints in a systematic fashion.

Theoretical development. The system of equations in (1) is highly underdetermined since both $f(t)$ and $p(t)$ are unknown. To avoid this problem, multichannel Wiener filtering is traditionally used to minimize the following misfit function:

$$
J_{1}=\|p(t)\|^{2}=\left\|y(t)-\sum_{j=1}^{N} f_{j}(t) * m_{j}(t)\right\|^{2} \rightarrow \min
$$

After some routine treatment in the least-squares sense, the minimization problem above yields the following linear system of equations:

$$
M^{\mathrm{T}} \mathrm{MF}=\mathrm{M}^{\mathrm{T}} \mathrm{Y}
$$

$\mathrm{M}$ is a matrix with $\mathrm{N}$ columns of vectors which are windowed seismic traces, $\mathrm{Y}$ is a vector whose elements are 
Figure 2. Inverted primary (dotted line) and true primary (solid line). The energy of inverted primary is always less than the true one (they are equal if and only if primary and multiple events are orthogonal). This

raises a serious problem in practice since the application of least-squares subtraction introduces "leakage" between primaries and multiples. (Modified from Spitz.)
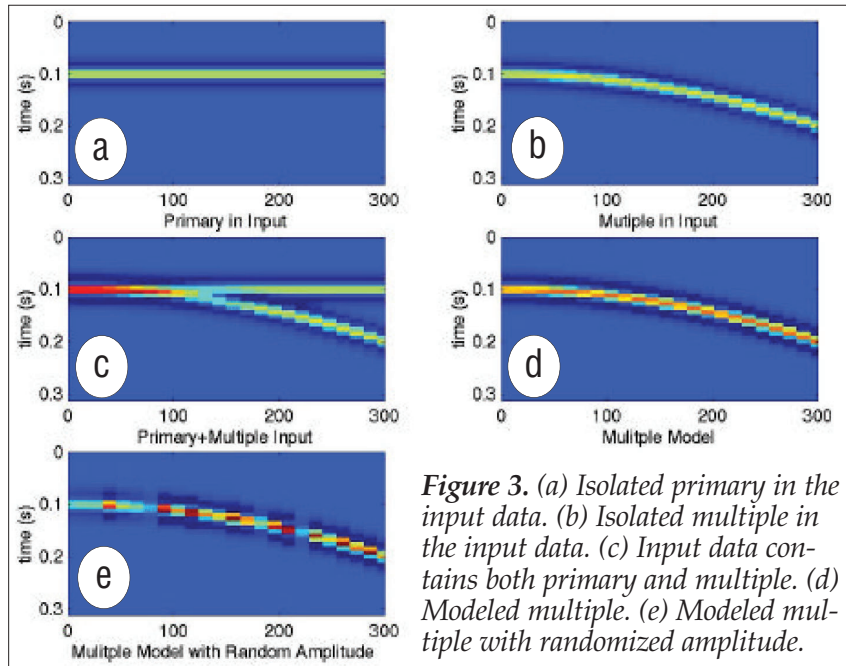

Figure 3. (a) Isolated primary in the input data. (b) Isolated multiple in the input data. (c) Input data contains both primary and multiple. (d) Modeled multiple. (e) Modeled mulMulitple Model with Random Amplitude tiple with randomized amplitude.

amplitude samples of the input trace within a sliding window, and F is the desired filter. The superscript $\mathrm{T}$ denotes the transpose of a matrix. Equation 3 often can be readily solved with well-established algorithms.

The key to the emergence of equation 3 as a solvable linear system of equations is the assumption that primaries and multiples have to be orthogonal. If so, dot multiplication of both sides of equation 1 by the multiples $\mathrm{m}(\mathrm{t})$ will produce equation 3 because, if $\mathrm{p}(\mathrm{t})$ and $\mathrm{m}(\mathrm{t})$ are orthogonal, their dot product is zero.

Figure 2 illustrates the orthogonal assumption underlying equations 2 and 3 . The original data vector minus the multiple model vector results in the primary reflection vector. The power-minimized solution of equation 2 is the dotted line that has the shortest norm.

However, in most cases, the orthogonal assumption is not valid, and (as shown in Figure 2) the dotted line differs from the solid arrow $\mathrm{p}$. To resolve this fundamental problem, we revisit equation 1 and propose an alternative solution. The new solution, instead of minimizing the power of primaries (the $\mathrm{J}_{1}$ ), minimizes the following function (Claerbout, 1992):

$$
\mathbf{J}_{2}=\left\|\mathrm{y}(\mathrm{t})-\mathrm{p}(\mathrm{t})-\sum_{\mathrm{j}=1}^{\mathrm{N}} \mathrm{f}_{\mathrm{j}}(\mathrm{t}) * \mathrm{~m}_{\mathrm{j}}(\mathrm{t})\right\|^{2} \rightarrow \min
$$

Thus, our key requirement is that the nonpredictable energy in the data (the result after applying PEF) be as small as possible. That is

$$
\begin{aligned}
& \mathrm{J}_{3}=\|\operatorname{PEF}(\mathrm{p}(\mathrm{t}))\| \rightarrow \min \\
& \mathrm{J}_{4}=\|\operatorname{PEF}(\mathrm{m}(\mathrm{t}))\| \rightarrow \min
\end{aligned}
$$

The misfit functions in equation 4 can be minimized
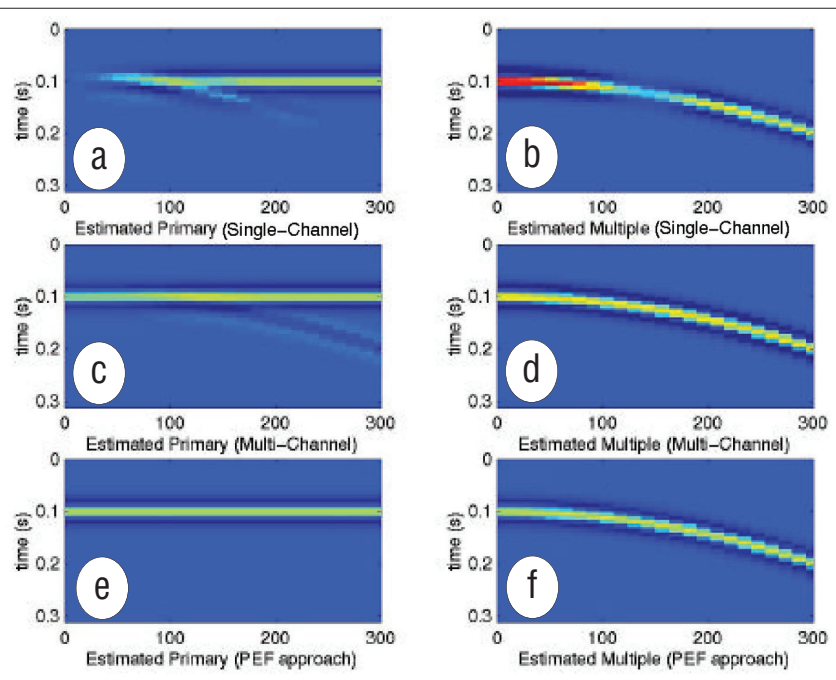

Figure 4. Inverted primary (left) and multiple (right) with single-channel (top), multichannel (middle), and SIMP methods (bottom). The modeled multiple used in the inversion is shown in Figure $3 d$. The desired right answers are Figures $3 a$ and $3 b$, respectively.

together. However, $\mathrm{J}_{3}$ and $\mathrm{J}_{4}$ do not necessarily need to be enforced simultaneously and they can be used selectively based on the data. The PEF can be determined using various ways. The simplest PEF is the derivative operator, $(d / d x)$, which means that the seismic events have minimal discontinuities laterally. Improved PEFs can be determined using other geologic discriminants. For instance, if we know the dip of a multiple generator, we can minimize the directional derivative of the multiples along the dip direction. This can be done to primaries as well.

As an example, let us write the system of equations derived from the optimization problem (4a) and (4b). Dropping $\mathrm{J}_{3}$ and using the $\mathrm{R}$ (roughness) operator to represent the PEF, we get:

$$
\left[\begin{array}{cc}
\mathrm{I} & \mathrm{M} \\
\mathrm{R} & 0
\end{array}\right]\left[\begin{array}{l}
\mathrm{P} \\
\mathrm{F}
\end{array}\right]=\left[\begin{array}{l}
\mathrm{Y} \\
0
\end{array}\right]
$$

Of course, a more general set of equations can be obtained if we take into account the $\mathrm{J}_{4}$ term. The optimization problem of $\mathrm{J}_{2}, \mathrm{~J}_{3}$, and $\mathrm{J}_{4}$ can be rewritten in the form of the matrix:

$$
\left[\begin{array}{cc}
\mathrm{I} & \mathrm{I} \\
\mathrm{R} & 0 \\
0 & \mathrm{Z}
\end{array}\right]\left[\begin{array}{c}
\mathrm{P} \\
\mathrm{M}
\end{array}\right]=\left[\begin{array}{l}
\mathrm{Y} \\
0 \\
0
\end{array}\right]
$$

here, $\mathrm{R}$ and $\mathrm{Z}$ represent the PEFs of primaries and multiples respectively. $\mathrm{R}$ and $\mathrm{Z}$ can be derived from a priori knowledge, including (but not limited to) modeled multiples, modeled primaries, and/or geologic models derived from seismic interpretation. With equation 6, we invert simultaneously for both multiples and primaries using geologic constraints. This is the reason the acronym SIMP (simultaneous inversion of multiple and primary) is adopted for this method, although we can also invert for shaping filters (Figure 1b) with primaries simultaneously.

Synthetic examples. A series of numerical examples supports the predictions of the theory. In all the synthetics, we use the simplest PEF operator (i.e., $\mathrm{d} / \mathrm{dx}$ ) and equation 5.

Figure $3 \mathrm{c}$ shows a synthetic data set consisting of a primary (Figure 3a) and a multiple event (Figure 3b). The robustness of the proposed SIMP inversion is tested using 


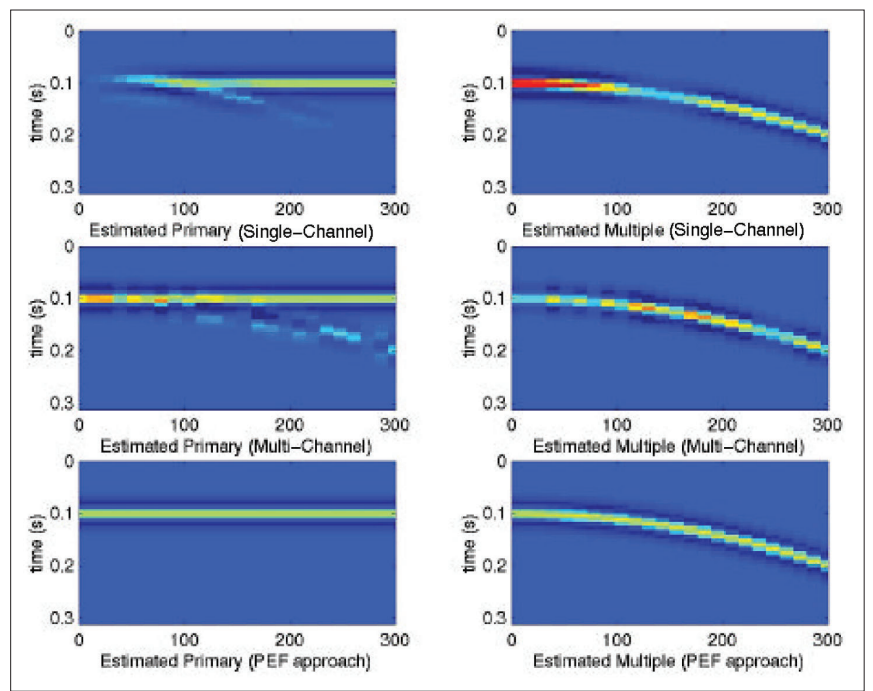

Figure 5. Inverted primary (left) and multiple (right) with single-channel (top), multichannel (middle), and SIMP methods (bottom). The modeled multiple used in the inversion is shown in Figure 3e, which contains random amplitudes. The desired right answers are Figures $3 a$ and $3 b$, respectively.
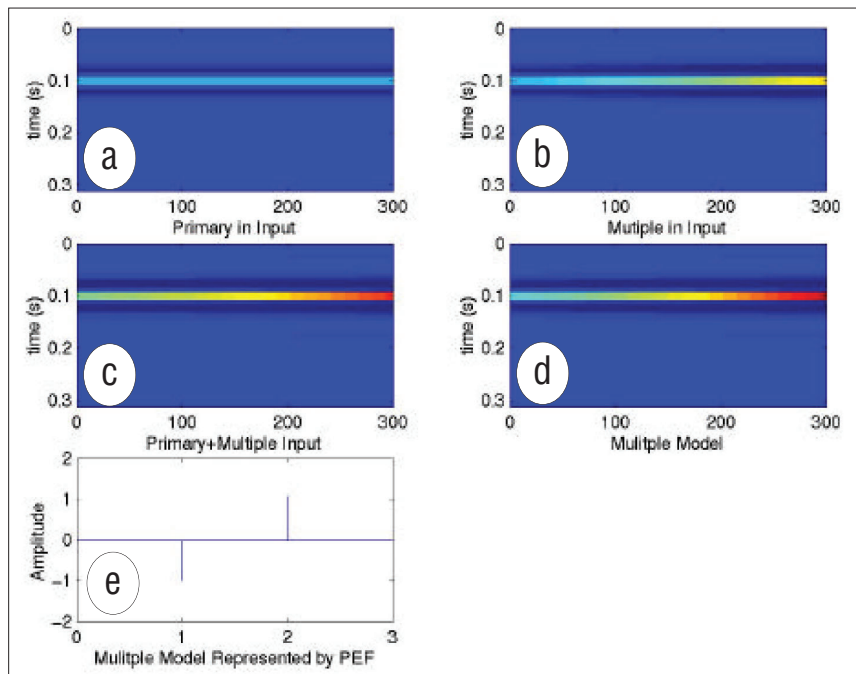

Figure 6. (a) Single primary in the input data. (b) Single multiple in the input data. (c) Input data contains both primary and multiple. Notice the complete overlap of the events. (d) Modeled multiple. (e) PEF of modeled multiple with samples $(-1,1.05)$.

two modeled multiples. Figure 3d shows a multiple model obtained by multiplying the true amplitude of Figure $3 \mathrm{~b}$ by a scalar factor. Figure $3 \mathrm{e}$ is the same as Figure $3 \mathrm{~d}$ except that the amplitude is now modulated randomly. Figure 4 shows the inverted primary and multiple events using single-trace, multichannel, and SIMP approaches, respectively. In this example, the simple modeled multiple in Figure $3 \mathrm{~d}$ is used as input for the inversion. Notice the exact estimation of both events offered by the SIMP algorithm. Figure 5 is the same as Figure 4, but now the modeled multiple used is the one in Figure 3e. This is a more difficult case since the amplitude of the modeled multiple is far from the true one. Again the SIMP results are superior than those obtained via the single and multichannel predictions.

Figure 6 shows another synthetic data set consisting of a primary (Figure 6a) and a multiple event (Figure 6b). This is a tough model because the multiple and primary completely overlap. Figure $6 \mathrm{~d}$ shows a multiple model that is a scaled version of Figure $6 \mathrm{~b}$. Figure $6 e$ shows the PEF of the

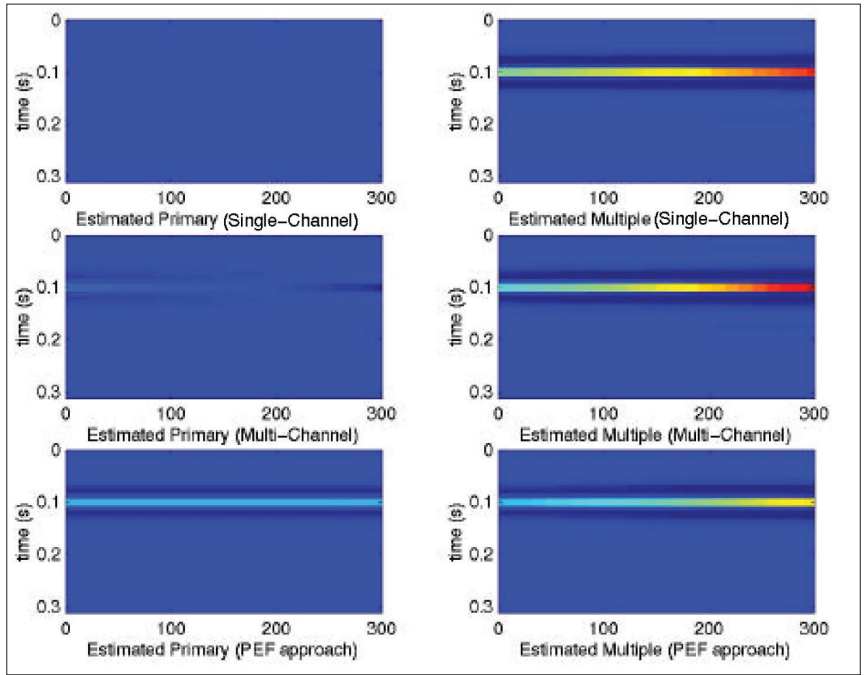

Figure 7. Inverted primary (left) and multiple (right) with single-channel (top), multichannel (middle), and SIMP methods (bottom). The modeled multiple used in the inversion is in Figure $5 d$. The desired right answers are Figures $5 a$ and $5 b$, respectively.
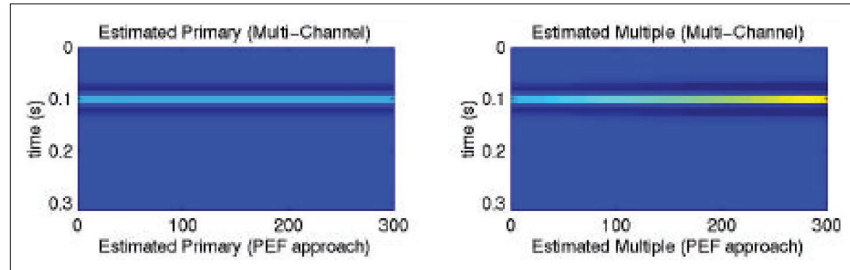

Figure 8. Inverted primary (a) and multiple (b) with SIMP method. The PEF of the modeled multiples (Figure 6e) is used in the inversion.

modeled multiple. The PEF can be derived from either the modeled multiple (e.g., through an fx-deconvolution approach) or from some type of geologic model (e.g., the dip of the multiple generator).

Figure 7 shows the inverted primary and multiple using single-trace, multichannel, and SIMP approaches, respectively. In this example, the simple modeled multiple (Figure $6 \mathrm{~d}$ ) is input for the inversion. Comparison with the right answers (Figures 6a and 6b) show that the SIMP approach produced almost perfect results while both conventional methods failed. Figure 8 shows the results of SIMP inversion using the PEF of the modeled multiple (Figure 6e). Again the recovered primary and multiple events are almost perfect. In this example, the more general SIMP method (Figure 1c) has been adopted with no direct information pertinent to multiple prediction and wavelet.

Summary. A concise theoretical formulation for direct estimation of primaries and multiples is presented that avoids the orthogonality assumption of the least-squares adaptive subtraction. Thus, it has the potential to minimize the "leakage" of primary energy when there is considerable crosstalk between primaries and multiples. Basically, the "processing" approach of the conventional least-squares subtraction is replaced with an "inversion" type algorithm. Its only assumption is the predictability of primary and multiple events. Additional a priori geologic knowledge (dip of multiple generator, regional trend of primaries, etc.) can be incorporated in the proposed inversion algorithm to achieve more accurate results. SIMP is not sensitive to amplitude errors in the modeled multiples as shown in Figure 5 where the amplitudes of predicted multiples are randomized. (Continued on $p$. 891) 
(Luo, from p. 818)

However, time errors in predicted multiple models can be handled if the SIMP II method is used as shown in Figure 8.

Suggested reading. "Estimation of multiple scattering by iterative inversion: Part I theoretical considerations" by Berkhout and Verschuur (GEOPHYSICS, 1997). Earth Soundings Analysis: Processing versus Inversion by Claerbout (Blackwell Scientific Publications, 1992). "Adaptive subtraction of multiples with the L-1 norm" by Guitton and Verschuur (DELPHI, XIII, 2002). "Surface-related multiple elimination on land seismic dataStrategies via case studies" by Kelamis and Verschuur (GEOPHYSICS, 2000). "Imaging the most bounce out of multiples" by Schuster (presented at multiple-reduction workshop of EAGE annual meeting, 2003). "Pattern recognition, spatial predictability, and subtraction of multiple events" by Spitz (TLE, 1999). "Multiple subtraction using an expanded multichannel filter" by Wang (GEOPHYSICS, 2003). TLE

Acknowledgments: We thank the Saudi Arabian Oil Company (Saudi Aramco) for encouraging this work and for permission to publish this paper. The previous work of Guus Berkhout, Jon Claerbout, Eric Verschuur, and Simon Spitz has helped and inspired us during this project. We also thank Mohammed Alfaraj for his critical review and comments.

Corresponding author:yi.luo@aramco.com 